\title{
Parameter Estimation of Damped Compound Pendulum Differential Evolution Algorithm
}

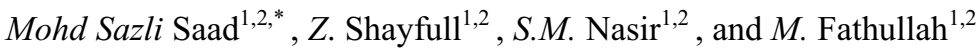 \\ ${ }^{1}$ Green Design and Manufacture Research Group, Center of Excellence Geopolymer and Green \\ Technology (CEGeoGTech), Universiti Malaysia Perlis, 01000 Kangar, Perlis, Malaysia \\ ${ }^{2}$ School of Manufacturing Engineering, Universiti Malaysia Perlis, Kampus Tetap Pauh Putra, 02600 \\ Arau, Perlis, Malaysia
}

\begin{abstract}
This paper present the parameter identification of damped compound pendulum using differential evolution algorithm. The procedure used to achieve the parameter identification of the experimental system consisted of input output data collection, ARX model order selection and parameter estimation using conventional method least square (LS) and differential evolution (DE) algorithm. PRBS signal is used to be input signal to regulate the motor speed. Whereas, the output signal is taken from position sensor. Both, input and output data is used to estimate the parameter of the ARX model. The residual error between the actual and predicted output responses of the models is validated using mean squares error (MSE). Analysis showed that, MSE value for LS is 0.0026 and MSE value for DE is $3.6601 \times 10-5$. Based results obtained, it was found that DE have lower MSE than the LS method.
\end{abstract}

\section{Introduction}

The pendulum is a relative mass object hung from the pivot point. Naturally, when the external force acts on it, it will swing back and forth with periodic motion. This kind of phenomena usually works due to the force of gravity. However, in this research the pendulum motion is powered by a driven propeller. When the propeller is driven by a specific amount of voltage, it will spin gradually hence produce a thrust force that moves the pendulum at certain angle position. This kind of driven pendulum can be seen in wide applications of multi-rotor helicopters which also known as drones. One of the vital parts to be focused on is the position control of the pendulum. This part is intrinsically unsteady systems, requiring exact control so as to fly. Therefore, in order to get a better control system, the physical system needs to be modeled accurately.

Therefore, in this research, system identification is employed in order to get an accurate model of damped compound pendulum. This due to the fact that, physical modeling of the real system is not as easy to acquire. Ones, needs to have a physical system about the system such as the dynamic behavior of a brushless DC motor, electronic speed controller (ESC) and aerodynamic forces and moment generated by the propeller installed on motor. Furthermore, ESC for the motor has it own dynamic property where a precise dynamic

\footnotetext{
*Corresponding author: sazlisaad@unimap.edu.my
} 
model is hard to derive using mathematical modeling. Parametric system identification is one of the simplest methods in system identification to be used for estimating the model from the actual input output experimental data. In practice, parametric estimation requires of three main steps, model structure selection, estimation of the model parameters and validation test. Many well-developed techniques, for instance, least square, maximum likelihood and instrumental variable.

Recently, nature inspired algorithms have become potential candidates to many parameter estimation techniques. Among them, differential evolution (DE) has been widely used in system identification and controller optimization. The prominent advantages of this technique is it can works effectively without require any search space and to be differentiable or continuous. Many researchers attempt to study the performance of DE in optimizing processes. Wenjuan $\mathrm{Gu}$ et. al (2016) utilized hybrid artificial bee colony algorithm combined with differential evolution to optimise the estimate parameters of the unknown fractional-order memristor-based chaotic systems. Results revealed that the unknown chaotic system can be estimated effectively with minimum residual error and higher convergence accuracy [1]. Ashik and Shahid (2016) studied the performance of DE in searching for the optimum proportional intergral (PI) controller parameters to track small variations in stand-alone solid oxide fuel cell (SOFC) system load current. The objective function was formulated based on the minimization of an eigenvalue in such a way that the error between the actual and desired eigenvalues were reduced. Comparative study showed that show that the performance of DE is superior than those of particle swarm optimization (PSO) and invasive weed optimization (IWO) [2]. Leandro et. al (2015) improved the original differential evolution (DE) in tuning the discrete VSC parameters for applied in CSTR plant. Results showd that discrete VSC parameters tuned by improve DE produced fast setpoint tracking and good disturbance rejection [3].

Over the years, parametric modeling with differential evolution (DE) algorithm has been widely used several engineering plant or system. Many researchers have employed the algorithms to estimate the unknown parameters in a parametric model structure of a particular system. The estimated model was found to be more accurate and effective than the conventional methods. AL-Khafaji and Darus (2014) applied differential evolutionary techniques (DE) and particle swarm optimization technique (PSO) to estimate the parametric model of flexible single-link manipulator system. The identified model was conducted based on the input output data from the simulation study using finite element method (FDM) based on Lagrangian approach. Mean squared error (MSE), correlation tests and one step ahead (OSA) validation test showed that DE performed better than PSO [4]. Sazli et. al (2012) proposed an alternative approach to search for the optimum parameter for the linear parametric structure controller to suppress the vibration of a flexible beam system. The objective function of the controller is the minimum value of mean square error. Simulation result depicted a sufficient vibration reduction with optimum controller setting [5]. Yousefi et. al (2008) identified the parametric model for electro hydraulic servo systems (EHSS) utilizing a novel evolutionary algorithms in particular differential evolution. The optimisation procedure was intended to seek for a global minimum in terms of mean square error (MSE) on a non-linear constraint functions with boundary limits of variables of the unknown parameters of a servo-hydraulic system. Rather than having a quick convergence profile, DE algorithm also exhibited a a good agreement between the predicted and measured value. The validity of the obtained model is guaranteed[6]. Swayamsiddha et. al (2015) investigated the performance of differential algorithm (DE) for nonlinear multiple input multiple output (MIMO) system. Convergence profile indicated that, the proposed algorithm able to prevent from trapping at local minima which cause a premature settling of the model parameters. Furthermore, with this result, it was assured that this technique can be used to solve for predicting model of real system [7]. Zakaria et. 
al (2015) employed multi-objective optimization differential evolution (MOODE) to estimate the dynamic model of palm oil biodiesel engine. There were two objective function aims to be minimised, the number of terms used model structure and the mean square error between measured and estimated outputs. Validation test has shown that the proposed algorithm accurately search for the optimal parameter of the estimated model [8]. From the overall study, it is assured that DE able to find the optimal solution for various applications. Apart from that, DE is useful, reliable and offer better solution in the fields of control system and particularly in system identification.

This paper attempts to estimate the model for motorized-propeller pendulum via the experimental approach using DE as a tool for model optimisation. The input output data is collected from the experimental rig comprise of input voltage to regulate the motor speed and the output voltage taken from the position sensor. Then, the simple model structure is selected i.e. ARX model structure. The model parameters is carried out using DE algorithm to find the best estimated model that accurately represent the actual system. Finally, the obtained model is validated using mean square error and one step ahead (OSA) predicton. At the end of the study, the performance of DE is compared with conventional technique namely least square (LS).

\section{Experimental setup}

In the present work, the real time interacting and non-interacting fabricated system was used for collecting the input, output data. In this part will explain the parameter and function for each component/device. The setup consists of hardware and software. The hardware (Figure 1) use in this project is DC power supply, Brushless speed controller, Arduiono , Data Acquisition card National Instrument, Automatic voltage regulator, Motorized Propeller, potentiometer, and pendulum apparatus. The set-up is intended to study dynamic response of single input and single output for motorized propeller pendulum. Software use for this project to the system identification are Matlab and Simulink.

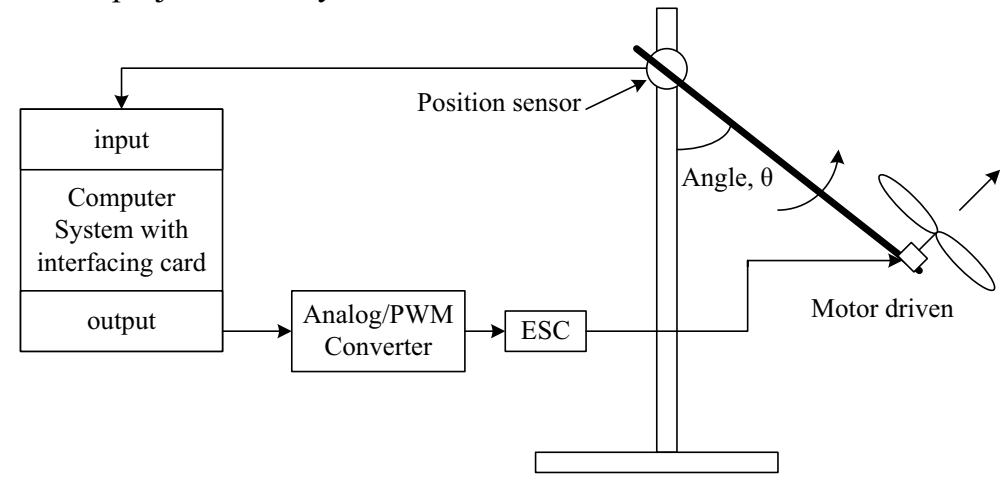

Fig. 1. Schematic diagram for motorized propeller system.

Figure 2 shows the process of system identification. Initially, data collection is adopted. The input of the system was analog output to the motor and the output was the input voltage from the position sensor. Based on the acquired input-output data, the model parameter is estimated until minimum error, $\mathrm{e}(\mathrm{t})$ is reached. The parameter of estimated model is determined using the differential evolution algorithm and compared with least square (LS) [9]. The performance of the estimated model is measured using mean square error (MSE). 


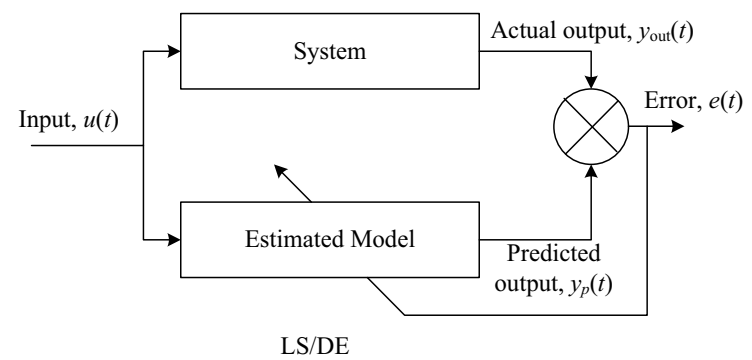

Fig. 2. The system and the model.

\section{System identification}

The system identification is the process to get the system model through the data inputoutput system (stimulus-response). The data input and output can be obtained through the experiments, simulations or directly collected from the factory. After the model of the physical system is obtained, it can be used to solve a variety of problems such as, for controlling physical systems or to predict the behaviour under different operating conditions [10].

The goal Identification System is a mathematical model based on experimental data of dynamic systems. Model should be solid and sufficient in respect of the purpose it is intended for. Identification system more systematic information from experimental observation is a key aspect of any scientific work. The information obtained led to the establishment of model systems considered. Models such as some form of pattern that explains the observed experimental results and justify predictions of future system response to be made.

In system identification there are three types of white-box model, Grey-box and black box. The most suitable identification for this project is black-box identification [11]. Black box model assumes that the system is unknown and all model parameters can be adjusted regardless of the physical background. Type of system identification that is appropriate for this project is the black-box. Most system identification algorithms are using this type of system to obtain system model. Black-box identification are rarely used in real-life control.

\subsection{Model structure}

In this study, the chosen method for estimating the model of driven pendulum system is single input and single output Auto-regressive with eXogenous (ARX) input model structure. ARX model is the simpliest identification technique for black-box parametric model. This model is developed from a linear regression analysis which is very efficient in presenting the actual dynamic systems. Moreover, the solution is unique. In other words, the solution always satisfies the global minimum of the loss function [4]. The general equation of ARX model is shown in equation (1).

$$
y[k]=\frac{B(q)}{A(q)} u[k]+\frac{1}{A(q)} e[k]
$$

The transfer function form of this ARX model is:

$$
\frac{y_{n}}{u_{n}}=\frac{b_{1} q^{-1}+b_{2} q^{-2}+\ldots b_{r} q^{-r}}{1-a_{1} q^{-1}-a_{2} q^{-2}-\ldots-a_{m} q^{-m}}
$$

The model parameter, $a$ and $b$ are estimated using Differential evolution algorithm (DE) and compared with least square (LS) method. 


\subsection{Least squares (LS)}

The least square (LS) parameter estimator is one of the conventional estiomation algorithm. It was discovered by Karl Friedrich Gauss in 1975 for astronomical computation purposed. This method works based on minimizing the residual error between actual and estimated outputs. This method is used because it is easy to implement and suitable for higher order linear system [12]. The least squares method is also known as linear regression and equation error method.

The LS method estimates the model parameter, $\theta(k)$, denoted by $\hat{\theta}(k)$, such that it minimize the sum of the squares of the residual, $E(k)$. Parameter, $\theta(k)$ is obtained by minimizing the cost function in equation (3).

$$
J=\frac{1}{2} \sum_{K=1}^{N}[E(K)]
$$

Consider the mathematical model written in the regression model in equation (4):

$$
\begin{aligned}
& y(k)=x_{1}(k) \theta_{1}+x_{2}(k) \theta_{2}+\ldots+x_{i}(k) \theta_{i}=\left[\begin{array}{llll}
x_{1}(k) & x_{2}(k) & \ldots & x_{i}(k)
\end{array}\right]\left[\begin{array}{c}
\theta_{1} \\
\theta_{2} \\
\vdots \\
\theta_{i}
\end{array}\right]= \\
& \varphi^{t}(k) \underline{\theta}
\end{aligned}
$$

Model parameter, $\theta(k)$ is determined such that the output calculated the right-hand side of equation (4) matches as closed as possible with the measured $\mathrm{y}(k)$ in the sense of least squares, by minimizing:

$$
\begin{aligned}
& J=\frac{1}{2} \sum_{K=1}^{N}\left[y(k)-\varphi^{T}(k) \underline{\theta}\right] \\
& J=\frac{1}{2} E(N)^{T} E(N)
\end{aligned}
$$

The variables in equation 3.2 are given by:

$$
\begin{aligned}
& E(N)=Y(N)-\hat{Y}(N) \\
& =Y(N)-\phi(N) \underline{\theta} \\
& =\left[\begin{array}{llll}
\varepsilon(1) & \varepsilon(2) & \ldots & \varepsilon(N)
\end{array}\right] \\
& Y(N)^{T}=\left[\begin{array}{llll}
y(1) & y(2) & \ldots & y(N)
\end{array}\right] \\
& \phi(N)=\left[\begin{array}{c}
\varphi^{T}(1) \\
\varphi^{T}(2) \\
\vdots \\
\varphi^{T}(N)
\end{array}\right]
\end{aligned}
$$

$J$ is minimized by setting:

$$
\frac{\mathrm{d} J}{\mathrm{~d} \theta}=-\phi^{T}(Y-\phi \theta)=0
$$

Finally the estimated parameter is given by:

$$
\hat{\theta}=\left[\phi(N)^{T} \quad \phi(N)^{-1}\right] \phi(N)^{T} Y(N)
$$

Where,

$k=$ discrete time index

$u(k) \quad=$ system input at discrete-time $k$ 
$y(k) \quad=$ measured system output at discrete-tike $k$

$\underline{\theta}(k) \quad=$ unknown parameter vector to be estimated

$\underline{\hat{\theta}}(k) \quad=$ estimate of $\theta(k)$

$\bar{\varphi}(k) \quad=$ vector of input and output signal, and possibly their delayed signals

$\varepsilon(k) \quad=y(k)-\bar{y}(k)=$ residual

$\hat{y}(k) \quad=$ estimated output signal.

Equation (7) is used to find the estimated parameter for batch processing of LS method. The method can be used to find the relationship between two variable of linear system.

\subsection{Differential evolution (DE) algorithm}

Differential Evolution (DE), proposed by Storn and Price, is metaheuristic optimisation method to seek for global optimisation of a particular feasible solutions. It has a capabilities to deal with various problem ranging from linear system, nonlinear system, handling nondifferentiable, and multi-modal objective functions, with easier selection of control parameters. DE works similarly with a standard evolutionary algorithm (EA). However, there is a bit different from other EA in the mutation and recombination phases. DE uses weighted differences between solution vectors to change the population [13]. Figure 3 shows the flow of DE operations.

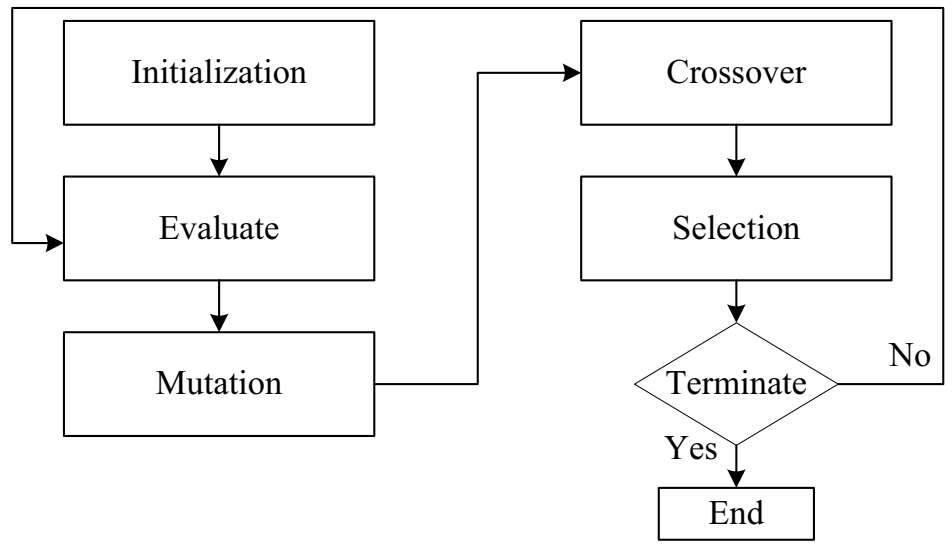

Fig. 3. Flow chart for DE.1

\subsubsection{Initialization}

Initialization process is performed to set appropriate parameter setting such as number of generation mutation rate, differential factor, and boundary of each solution vector. All the vector population and its fitness value randomly initialized in the given upper \& lower bound.

\subsubsection{Mutation}

Three set of vector population is randomly selected, and the mutant vector is evaluated using equation (8). The difference between two vectors is multiply with its mutant factor, $F$. 
A mutant vector is generated by:

$$
v_{j, G+1}=x_{r 1, G}+F \cdot\left(x_{r 2, G}-x_{r 3, G}\right)
$$

where the three distinct vectors $\mathrm{x}_{\mathrm{r} 1}, \mathrm{x}_{\mathrm{r} 2}$ and $\mathrm{x}_{\mathrm{r} 3}$ randomly chosen from the current population other than vector $\mathrm{x}_{\mathrm{j}, \mathrm{G}}$.

\subsubsection{Crossover}

Upon approaching the mutation operation, The trial vector $v_{i, G+1}$ is developed via crossover process between the target vector and the mutant vector, $X i, G$ using equation (18). Crossover is done in order to increase the diversity of the perturbed parameters for each individual in the population [7].

$$
u_{j, i, G+1}=\left\{\begin{array}{ll}
v_{j, i, G+1} & \text { if } \text { rand } \leq C R \\
x_{j, i, G} & \text { if } \text { rand }>C R
\end{array}\right\}
$$

\subsubsection{Selection}

After the mutation and crossover operations, the fitness value from the trial vector and target vector is compared. The best fitness value is reserved for the next generation.

\section{Results and discussion}

In this project, the experiment was carried out to compare the value of mean square error (MSE) for least squares (LS) and differential evolution (DE) algorithm. Input-output data from the actuator and position sensor are used for parameter estimation in Least squares (LS) algorithm and differential evolution (DE) algorithm. Figure 4 and 5 show position sensor voltage signal and PRBS actuator signal respectively.

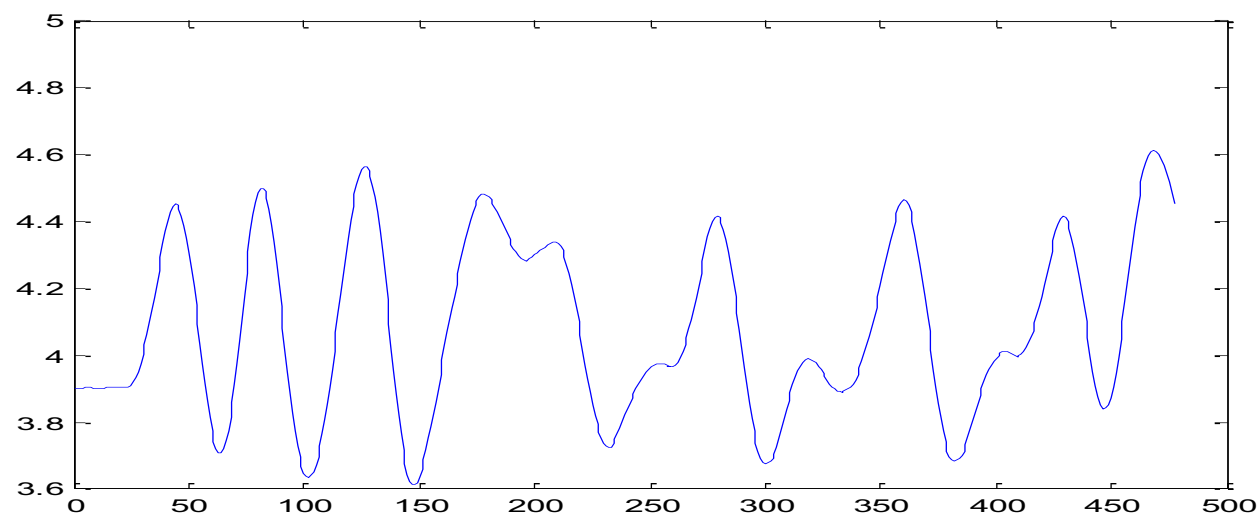

Fig. 4. Output voltage from potentiometer 


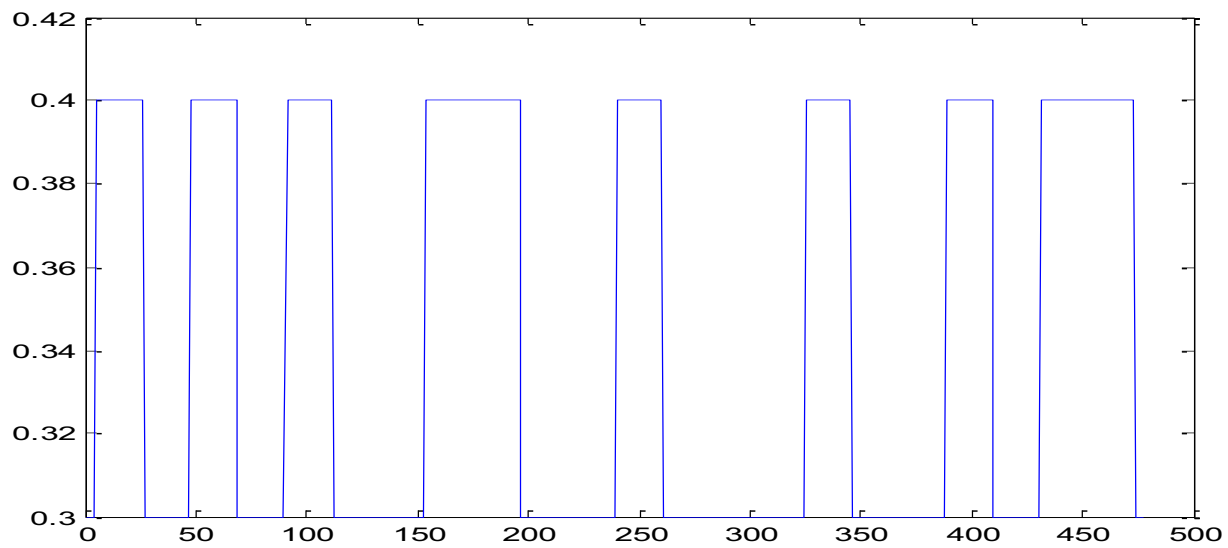

Fig. 5. PRBS input voltage to ESC

Initially for DE, the control parameter such as number of population, NP and generation, GEN is set to 30 and 100 respectively. The experiment was carried out to investigate the value of MSE by changing the crossover rate, CR between 0.1 to 1 .

Table 1. The value for model parameter, crossover and MSE for NP=30.

\begin{tabular}{|c|c|c|c|c|c|c|}
\hline \multicolumn{4}{|c|}{ Beta } & \multirow{2}{*}{ CR } & \multicolumn{2}{c|}{ MSE(Output) } \\
\cline { 1 - 3 } \cline { 6 - 7 } a1 & $\mathrm{a} 2$ & $\mathrm{~b} 1$ & $\mathrm{~b} 2$ & & Train & Test \\
\hline-1.7556 & 0.7922 & 0.0186 & -0.0121 & 0.1 & $5.8047 \times 10^{-4}$ & $3.3974 \times 10^{-4}$ \\
\hline-1.8379 & 0.8591 & -0.0218 & 0.0120 & 0.2 & $2.3507 \times 10^{-4}$ & $2.5269 \times 10^{-4}$ \\
\hline-2.0206 & 1.0414 & -0.0016 & 0.0088 & 0.3 & $8.8214 \times 10^{-5}$ & $9.6176 \times 10^{-5}$ \\
\hline $\mathbf{- 1 . 9 9 5 6}$ & $\mathbf{1 . 0 1 1 6}$ & $\mathbf{- 0 . 0 0 2 1}$ & $\mathbf{0 . 0 0 3 0}$ & $\mathbf{0 . 4}$ & $\mathbf{3 . 2 0 5 9} \times 10^{-5}$ & $\mathbf{3 . 8 3 3 8 \times 1 0 ^ { - 5 }}$ \\
\hline-1.9713 & 0.9877 & -0.0026 & 0.0042 & 0.5 & $3.1864 \times 10^{-5}$ & $3.8973 \times 10^{-5}$ \\
\hline-1.9839 & 1.0013 & -0.0012 & 0.0016 & 0.6 & $3.0536 \times 10^{-5}$ & $4.2412 \times 10^{-5}$ \\
\hline-1.9815 & 0.9992 & -0.0025 & 0.0031 & 0.7 & $3.0261 \times 10^{-5}$ & $4.2477 \times 10^{-5}$ \\
\hline-1.9804 & 0.9980 & -0.0021 & 0.0028 & 0.8 & $3.0223 \times 10^{-5}$ & $4.2179 \times 10^{-5}$ \\
\hline-1.9806 & 0.9982 & -0.0021 & 0.0028 & 0.9 & $3.0222 \times 10^{-5}$ & $4.2178 \times 10^{-5}$ \\
\hline-1.9806 & 0.9982 & -0.0021 & 0.0028 & 1.0 & $3.0222 \times 10^{-5}$ & $4.2178 \times 10^{-5}$ \\
\hline
\end{tabular}

Table 2. The value for model parameter, crossover and MSE for NP=100.

\begin{tabular}{|c|c|c|c|c|c|c|}
\hline \multicolumn{4}{|c|}{ Beta } & \multirow{2}{*}{ CR } & \multicolumn{2}{c|}{ MSE(Output) } \\
\cline { 1 - 1 } \cline { 6 - 7 } a1 & $\mathrm{a} 2$ & $\mathrm{~b} 1$ & $\mathrm{~b} 2$ & & Train & Test \\
\hline-1.9110 & 0.9466 & 0.0196 & -0.0202 & 0.1 & $2.2701 \times 10^{-4}$ & $2.3989 \times 10^{-4}$ \\
\hline-2.0314 & 1.0450 & -0.0102 & 0.0103 & 0.2 & $6.1928 \times 10^{-5}$ & $4.4968 \times 10^{-5}$ \\
\hline $\mathbf{- 1 . 9 6 1 4}$ & $\mathbf{0 . 9 7 2 5}$ & $\mathbf{- 0 . 0 0 4 1}$ & $\mathbf{0 . 0 0 3 8}$ & $\mathbf{0 . 3}$ & $\mathbf{4 . 9 1 8 4 \times 1 0 ^ { - 5 }}$ & $\mathbf{3 . 6 6 0 1 \times 1 0 ^ { - 5 }}$ \\
\hline-1.9440 & 0.9616 & -0.0035 & 0.0041 & 0.4 & $3.7794 \times 10^{-5}$ & $4.5543 \times 10^{-5}$ \\
\hline-1.9770 & 0.9935 & -0.0016 & 0.0026 & 0.5 & $3.0696 \times 10^{-5}$ & $3.9261 \times 10^{-5}$ \\
\hline-1.9851 & 1.0023 & -0.0006 & 0.0014 & 0.6 & $3.0644 \times 10^{-5}$ & $4.1630 \times 10^{-5}$ \\
\hline-1.9799 & 0.9974 & -0.0028 & 0.0033 & 0.7 & $3.0296 \times 10^{-5}$ & $4.2009 \times 10^{-5}$ \\
\hline-1.9805 & 0.9981 & -0.0021 & 0.0028 & 0.8 & $3.0222 \times 10^{-5}$ & $4.2178 \times 10^{-5}$ \\
\hline-1.9806 & 0.9982 & -0.0021 & 0.0028 & 0.9 & $3.0222 \times 10^{-5}$ & $4.2178 \times 10^{-5}$ \\
\hline-1.9806 & 0.9982 & -0.0021 & 0.0028 & 1.0 & $3.0222 \times 10^{-5}$ & $4.2178 \times 10^{-5}$ \\
\hline
\end{tabular}


From Table 3, when $\mathrm{NP}=100$, the lowest MSE (test) value is $3.6601 \times 10^{-5}$ at $\mathrm{CR}=0.3$ and for $\mathrm{NP}=30$ the lowest MSE value is $3.8338 \times 10^{-5}$ at $\mathrm{CR}=0.4$. Among these tests, the lowest value for MSE is $3.6601 \times 10^{-5}$ at $\mathrm{CR}=0.3$ with $\mathrm{NP}=100$. This is the best parameter for the ARX model to present the dynamic driven pendulum system.

Table 3. Value of Beta, CR, NP and MSE (test)

\begin{tabular}{|c|c|c|c|c|c|c|}
\hline \multicolumn{4}{|c|}{ Beta } & \multirow{2}{*}{ CR } & \multirow{2}{*}{ NP } & \multirow{2}{*}{ MSE(test) } \\
\hline a1 & $\mathrm{a} 2$ & $\mathrm{~b} 1$ & $\mathrm{~b} 2$ & & & \\
\hline-1.9956 & 1.0116 & -0.0021 & 0.0030 & 0.4 & 30 & $3.8338 \times 10^{-5}$ \\
\hline-1.9614 & 0.9725 & -0.0041 & 0.0038 & 0.3 & 100 & $3.6601 \times 10^{-5}$ \\
\hline
\end{tabular}

\subsection{Comparison between least squares (LS) with differential evolution (DE) algorithm.}

The table 4 proved that the DE method produced the lowest MSE value $3.6601 \times 10^{-5}$, and parameter $a$ and $b$ values are $\mathrm{a}_{1}=-1.9614, \mathrm{a}_{2}=0.9725, \mathrm{~b}_{1}=-0.0041$ and $\mathrm{b}_{2}=0.0038$. From Figure (99) it is clearly depicted that differential evolution (DE) algorithm performed better than least square (LS) algorithm.

Table 4. Comparison between least squares (LS) with differential evolution (DE) algorithm.

\begin{tabular}{|c|r|r|r|r|c|c|}
\hline \multirow{2}{*}{ Method } & \multicolumn{4}{|c|}{ Beta } & \multicolumn{2}{c|}{ MSE } \\
\cline { 2 - 7 } & $\mathbf{a 1}$ & $\mathbf{a 2}$ & $\mathbf{b 1}$ & $\mathbf{b 2}$ & Train & Test \\
\hline $\begin{array}{c}\text { Least } \\
\text { Squares }\end{array}$ & 2.9196 & 1.9717 & -0.0065 & 0.0088 & 0.0057 & 0.0026 \\
\hline $\begin{array}{c}\text { Differential } \\
\text { Evolution }\end{array}$ & -1.9614 & 0.9725 & -0.0041 & 0.0038 & $4.9184 \times 10^{-5}$ & $3.6601 \times 10^{-5}$ \\
\hline
\end{tabular}

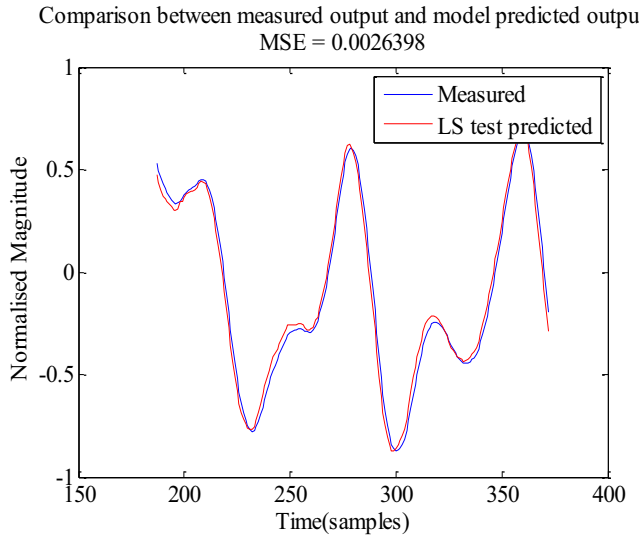

(a)

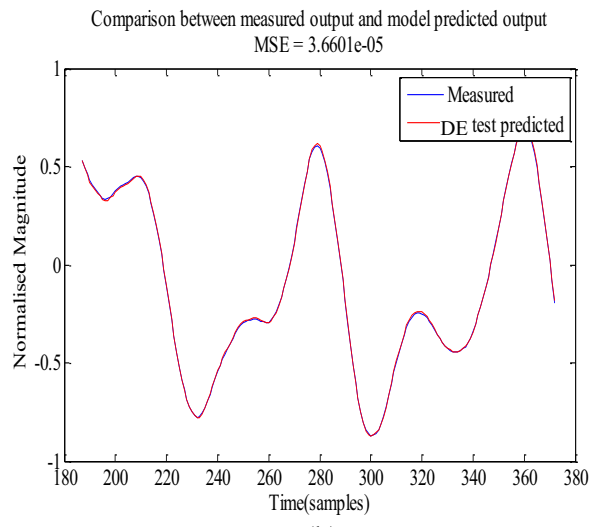

(b) 


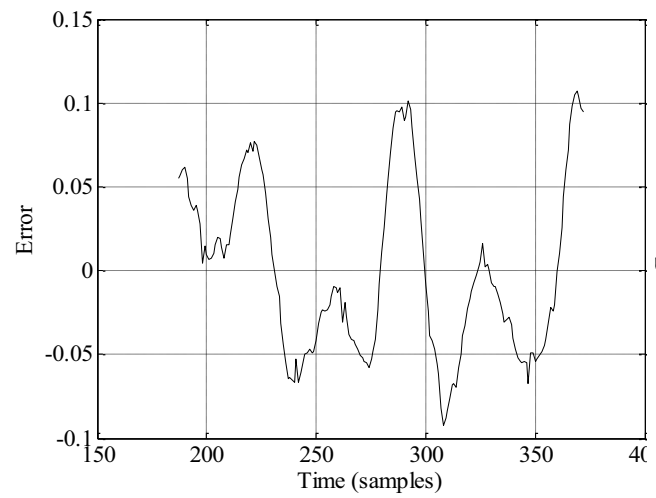

(c)

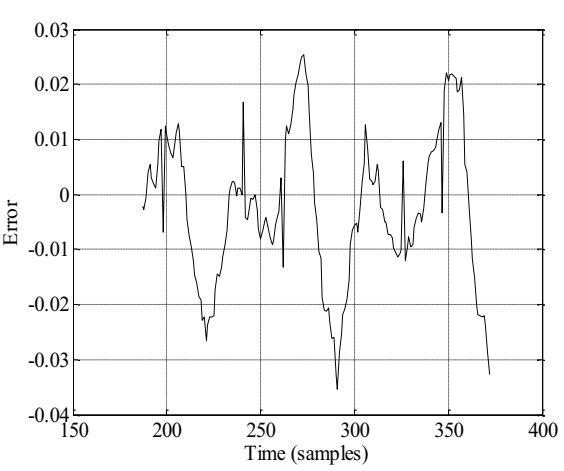

(d)

Fig. 2. Comparison between LS and DE. (a) actual and predicted output using LS, (b) actual and predicted output using DE, (c) Error between actual and predicted output using LS, (d) Error between actual and predicted output using DE

The transfer function for the ARX model using differential evolution algorithm is shown in equation (10).

$$
\frac{y(t)}{u(t)}=\frac{-0.0041 z^{-1}+0.0038 z^{-2}}{1+-1.9614 z^{-1}+0.9725 z^{-2}}
$$

\section{Conclusion}

The estimate model for motorized-propeller pendulum was successfully developed using $\mathrm{DE}$ as a tool for model optimisation. The model parameters is carried out using DE algorithm to find the best estimated model that accurately represent the actual system. Validation test showed that DE is performed better than least square (LS). For future work, the identified model will be useful to study the controller performance.

The authors would like to express their gratitude to Universiti Malaysia Perlis and Universiti Teknologi Malaysia for providing facilities to conduct this research. This research was funded by the Ministry of Education Malaysia through Fundamental Research Grant Scheme No. FRGS 900300451.

\section{References}

1. W. Gu, Y. Yu, W. Hu, Nonlinear Dynam., 84, 779 (2016)

2. A. Ahmed, M. S. Ullah, SpringerPlus, 5, 1, (2016)

3. L. d. S. Coelho, H. V. H. Ayala, A. A. R. Coelho, Chem. Eng. Res. Des., 100, 248 (2015)

4. A. AL-Khafaji, I. Darus, Evolutionary algorithm for identification of a flexible singlelink system, WSEAS International Conference on Manufacturing Engineering, Automatic Control and Robotics (Kuala Lumpur, 2014) 
5. M. S. Saad, H. Jamaluddin, I. Z. M. Darus, World Academy of Science, Engineering and Technology, International Journal of Mechanical, Aerospace, Industrial, Mechatronic and Manufacturing Engineering, 6, 446 (2012)

6. H. Yousefi, H. Handroos, A. Soleymani, Mechatronics, vol. 18, 513 (2008)

7. S. Swayamsiddha, S. Behera, H. P. Thethi, Blind Identification of Nonlinear MIMO System Using Differential Evolution Techniques and Performance Analysis of Its Variants, International Conference on Computational Intelligence and Networks (CINE, 2015)

8. R. Ahmad, M. Z. Zakaria, A. M. Nor, H. Jamaluddin, WSEAS Transactions on Systems and Control, 9, 500 (2014)

9. J. McLellan, Pseudo random binary sequence (PRBS TESTING, 2005)

10. L. Ljung, System Identification (Prantice Hall, New Jersey, 1999)

11. M. Killian, B. Mayer, M. Kozek, Energy Build., 96, 175, (2015)

12. J. G. Turner, B. Samanta, Least Squares Estimation of Dynamic System Parameters using LabVIEW, Proceedings of IEEE, (2012)

13. G. Y. Li, M. G. Liu, Int. Conf. Adv. Comput. Theory Eng. Proc., 3, 153, (2010) 